\title{
Patterns and prognostic relevance of PD-1 and PD-L1 expression in colorectal carcinoma
}

\author{
Lik Hang Lee ${ }^{1}$, Marcela S Cavalcanti ${ }^{1}$, Neil H Segal ${ }^{2}$, Jaclyn F Hechtman ${ }^{1}$, Martin R Weiser ${ }^{3}$, \\ J Joshua Smith ${ }^{3}$, Julio Garcia-Aguilar ${ }^{3}$, Eran Sadot ${ }^{3}$, Peter Ntiamoah ${ }^{1}$, Arnold J Markowitz ${ }^{2}$, \\ Moshe Shike ${ }^{2}$, Zsofia K Stadler ${ }^{2}$, Efsevia Vakiani ${ }^{1}$, David S Klimstra ${ }^{1}$ and Jinru Shia ${ }^{1}$ \\ ${ }^{1}$ Department of Pathology, Memorial Sloan Kettering Cancer Center, New York, NY, USA; ${ }^{2}$ Department of \\ Medicine, Memorial Sloan Kettering Cancer Center, New York, NY, USA and ${ }^{3}$ Department of Surgery, \\ Memorial Sloan Kettering Cancer Center, New York, NY, USA
}

\begin{abstract}
Immune checkpoint blockade targeting the programmed death-1 (PD-1) pathway has shown efficacy in several types of cancers including mismatch-repair-deficient colorectal carcinoma. In some tumor types, programmed death-ligand 1 (PD-L1) expression detected by immunohistochemistry has shown utility as a predictive marker for response to anti-PD-1 therapies. This utility, however, remains to be determined in colorectal carcinoma. In addition, although tumor-infiltrating lymphocytes have been associated with better prognosis in colorectal carcinoma, the prognostic value of PD-1 expression in these lymphocytes and its interaction with PD-L1 expression still await investigation. To address these questions, we performed a pilot study to evaluate the patterns of PD-L1 and PD-1 immunohistochemical expression on colorectal carcinoma cells and their tumorinfiltrating lymphocytes, respectively. Using tissue microarray, we found that $5 \%$ (19/394) of colorectal carcinomas exhibited high tumor PD-L1 expression, and 19\% (76/392) had elevated numbers of PD-1-positive tumor-infiltrating lymphocytes. PD-L1 levels correlated with PD-1 levels $(P<0.001)$, and mismatch-repairdeficient tumors had significantly higher rates of high PD-L1 and PD-1 expression when compared with mismatch-repair-proficient tumors (18\% vs $2 \%$ and $50 \%$ vs $13 \%$, respectively; $P<0.001$ for both). Staining intensity was also stronger for both markers in mismatch-repair-deficient tumors. Furthermore, we observed that among patients with mismatch-repair-deficient colorectal carcinoma, PD-1/PD-L1 expression stratified recurrence-free survival in an inter-dependent manner: an association between high PD-1-positive tumorinfiltrating lymphocytes and improved recurrence-free survival $(P=0.041)$ was maintained only when the tumors had low-level PD-L1 expression $(P=0.006)$; patients whose tumors had both high PD-1-positive tumor-infiltrating lymphocytes and high PD-L1 expression had a significantly worse recurrence-free survival $(P<0.001)$. Thus, our results not only provide a foundation for further assessment of PD-L1 immunohistochemistry as a predictive marker for anti-PD-1 therapy in colorectal carcinoma, they also shed light on the prognostic impact of tumorinfiltrating lymphocytes in different subsets of mismatch-repair-deficient colorectal carcinomas.
\end{abstract}

Modern Pathology (2016) 29, 1433-1442; doi:10.1038/modpathol.2016.139; published online 22 July 2016

In recent years, targeted therapies using antibodies against immune checkpoints have shown promising results in the treatment of various malignancies including melanoma, lung carcinoma, renal cell carcinoma, and urothelial carcinoma. ${ }^{1-7}$ The rationale for such an approach lies in the fact that immune checkpoints, such as programmed death-1 (PD-1) and its ligand programmed death-ligand

Correspondence: Dr J Shia, MD, Member and Attending Pathologist, Memorial Sloan Kettering Cancer Center, Professor of Pathology, Weill Cornell Medical College, 1275 York Avenue, New York, NY 10065, USA.

E-mail: shiaj@mskcc.org

Received 25 April 2016; revised 18 June 2016; accepted 20 June 2016; published online 22 July 2016
1 (PD-L1), are an immune-inhibitory mechanism by which carcinoma cells evade antitumor immunity. Expression of PD-L1 can negatively regulate T-cell antitumor activity by binding to the PD-1 receptor on the $\mathrm{T}$ cells. Thus, it is not surprising that in many tumors showing response to anti-PD-1 therapy, the tumor cells show expression of PD-L1 when tested by immunohistochemistry. ${ }^{2,3,6-8}$

In colorectal carcinoma, the efficacy of immune checkpoint therapy has been limited. ${ }^{2,4,5,9,10}$ It was confirmed only recently that colorectal carcinomas with proficient mismatch-repair function, which account for up to $96 \%$ of all metastatic colorectal carcinomas, are unlikely to respond to anti-PD-1 therapy. In a phase 2 study, objective response 
to pembrolizumab, an anti-PD-1 antibody, was achieved only in patients with mismatch-repairdeficient colorectal carcinoma (4/10, 40\%) and not in patients with proficient mismatch-repair colorectal carcinoma (0/18, 0\%). ${ }^{10}$ A question that follows is whether among the mismatch-repair-deficient colorectal carcinomas, PD-L1 and/or PD-1 expression bear any informative value in predicting response to anti-PD-1 therapy. Toward addressing this question, it is important to first determine whether immunohistochemical detection of PD-L1 and PD-1 is feasible in colorectal carcinoma and what their staining patterns are. Thus far, data in these regards are sketchy and incomplete.

Tumor-infiltrating lymphocytes have long been associated with an improved outcome in patients with colorectal carcinoma. ${ }^{11}$ Notably, most colorectal carcinomas with significantly increased lymphocytes fall into the mismatch-repair-deficient subset. $^{12}$ In this subset, it is unclear to what extent mismatch-repair deficiency per se versus high number of lymphocytes or both contribute to the better prognosis observed. It is intriguing to note that recent data have indicated that not all mismatchrepair-deficient colorectal carcinomas have a good prognosis. ${ }^{13,14}$ Could such prognostic differences be related to the lymphocyte infiltration in the tumors? Do the levels of PD-1 or PD-L1 expression in lymphocytes and tumor cells affect the prognostic impact of the tumor-infiltrating lymphocytes?

With these considerations, we designed this pilot study with two objectives. The first was to obtain preliminary data that may serve as a foundation for further exploration of the utility of PD-1 and PD-L1 immunohistochemistry as clinically relevant measures of response to anti-PD-1 therapies in colorectal carcinoma. For this, we examined the PD-1 and PD-L1 immunohistochemical expression patterns in a large series of colorectal carcinomas encompassing all tumor stages. The second objective was to evaluate the prognostic association, independent of immune checkpoint blockade, of PD-1 and PD-L1 in colorectal carcinomas with and without mismatch-repair deficiency.

\section{Materials and methods}

\section{Patient and Tumor Characteristics}

Study cases consisted of colorectal carcinomas surgically resected at a single cancer center. Only cases with accessible tissue blocks and clinical follow-up were included. Hematoxylin and eosin (H\&E) sections of all cases were systematically reviewed for pathologic features including tumor histologic type, differentiation, tumor-infiltrating lymphocytes (scored 0-3 with 0 being $<1$ tumor-infiltrating lymphocyte per 10 high power fields, 1 being 1-15 per 10 high power fields, 2 being $>15$ but $<215$ per 10 high power fields, and 3 being 215 or more per 10 high power fields), and peritumoral lymphocyte aggregates (scored $0-2$ with 0 being none, 1 being a few often $<5$, and 2 being $>5$ ). Tumor location was defined as being left-sided if the tumor was located at or distal to the splenic flexure. Chart review was performed to obtain clinical data including age, sex, neoadjuvant therapy, follow-up intervals, and time to recurrence. The study was approved by the institutional review board.

\section{Tissue Microarray}

The ATA-27 automated arrayer (Beecher Instruments, Sun Prairie, WI, USA) was used to create the tissue microarrays with $0.6 \mathrm{~mm}$ punch size. Tissue microarrays were constructed from representative tumor blocks for each case. Three cores of different areas of the tumor were sampled from each tumor specimen. Cases with no analyzable tumor in the resulting tissue microarrays were excluded from final analysis. The minimal requirement for a case to be regarded as analyzable was the presence of at least 1 intact core.

\section{Immunohistochemical Analyses}

Immunohistochemistry for PD-1 and PD-L1 was performed on tissue microarray slides. For PD-1, we used a monoclonal antibody against PD-1 (clone NAT105, ready to use, Catalog \# 760-4895, Cell Marque) on the Ventana Benchmark Ultra (Ventana Medical Systems, Tucson, AZ, USA). After optimizing the immunohistochemical conditions, the slides were deparaffinized. Target retrieval was performed for 24 min using 'Cell Condition 1'. The incubation time for the primary antibody was $16 \mathrm{~min}$. The slides were washed, followed by incubation with the secondary antibody and final development with the 3,3'-Diaminobenzidine Map Kit (Ventana, Catalog \# 760-700) for $10 \mathrm{~min}$, and detected with Ventana Optiview (Ventana, Catalog \# 760-700).

For PD-L1 immunohistochemistry, we used a monoclonal antibody against PD-L1 (clone E1L3N, dilution 1:250; Cell Signaling Technology, Danvers, MA, USA) on the Leica's Bond III platform (Leica Biosystems, Buffalo Grove, IL, USA). The dilution factor of this antibody was optimized by using liver parenchyma as negative control and tonsil tissue as positive control as recommended. In addition to antibody concentration, we also optimized target retrieval buffer. After optimizing the immunohistochemical conditions, the slides were deparaffinized. Target retrieval was performed in standard Epitope Retrieval Solution 2 (Catalog \# AR 9640, pH 9.0) slightly basic buffer. The primary antibody was diluted by 1:100 with a final concentration of $0.2 \mu \mathrm{g} / \mathrm{ml}$. The incubation time for the primary antibody was $30 \mathrm{~min}$. The slides were washed, followed by incubation with the secondary antibody and final development with the 3,3'-Diaminobenzidine Map Kit (Leica Biosystems, Catalog \# DS9800) for $7 \mathrm{~min}$, and detected with Leica's Bond Polymer Refine (Leica Biosystems, Catalog \# DS9800). 

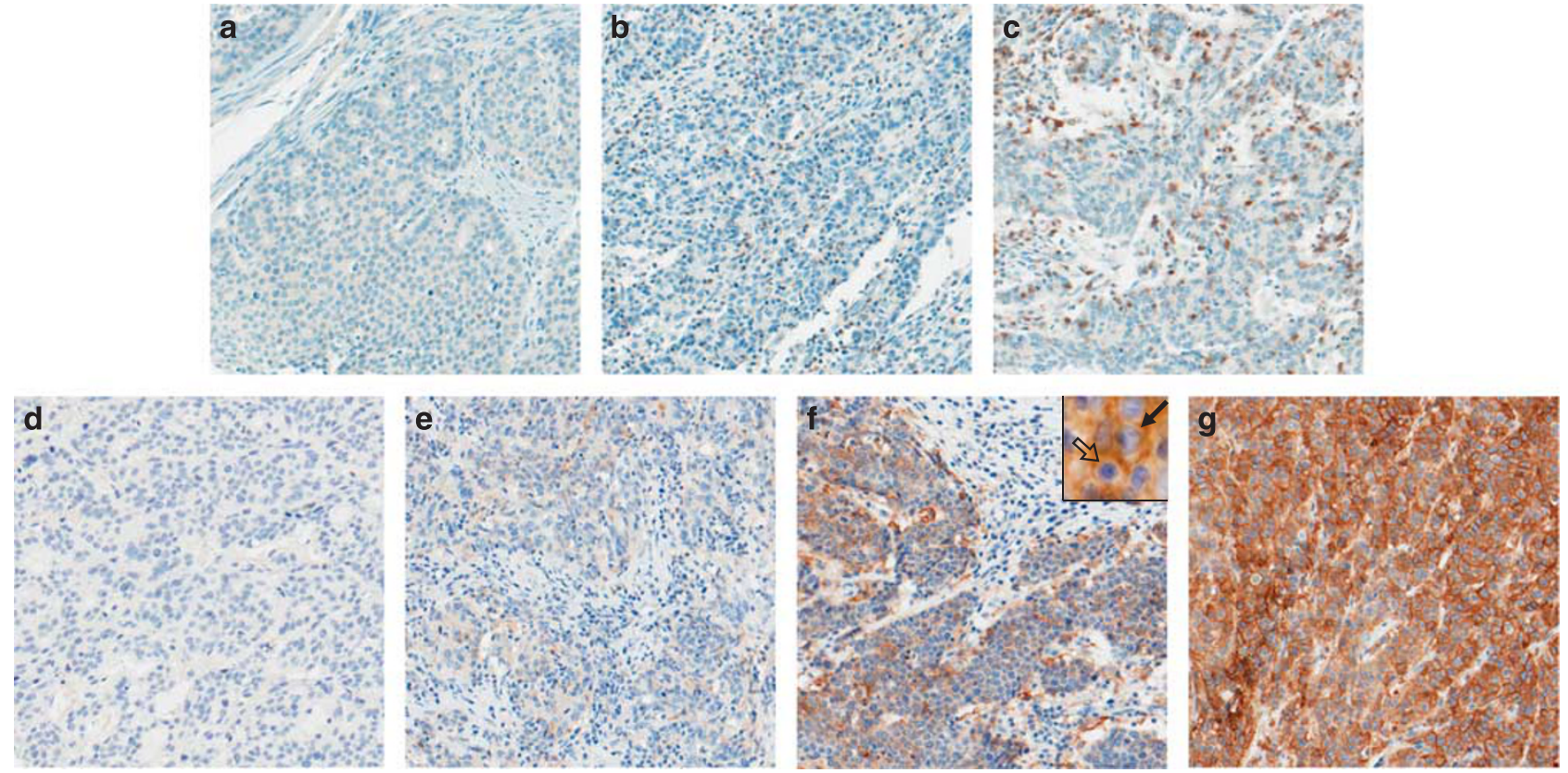

Figure 1 Immunohistochemical staining for PD-1 and PD-L1. The intensity of PD-1 expression on intraepithelial lymphocytes was scored as: 0 for no staining (a), 1+ for faint staining (b), and 2+ for moderate or strong staining (c). The intensity of PD-L1 expression on tumor cells was scored on a similar scale: 0 for no staining (d), 1+ for faint staining (e), and 2+ for moderate (f) or strong (g) staining. Staining of PD-L1 was seen in two patterns: staining primarily at the tumor-stroma interface and in both tumor cells and lymphocytes or immune cells (f), or diffuse labeling in the tumor cells (g). Inset in figure f highlights positive staining in both tumor cells (solid arrow) and lymphocytes (open arrow).

The stains for PD-1 and PD-L1 were scored as follows: (1) the amount of cells staining (percent of tumor cells staining for PD-L1, and the number of tumor-infiltrating lymphocytes per square millimeter of tumor staining for PD-1); and (2) the staining intensity using a scale of 0 to $2+$ : 0 for no staining, $1+$ for faint staining, and 2+ for moderate or strong staining (Figure 1).

Immunohistochemical staining for MLH1, PMS2, MSH2, and MSH6 was performed on the tissue microarrays using standard streptavidin-biotin-peroxidase procedure. Primary monoclonal antibodies used included MLH1 (clone G168-728, diluted 1:250, BD PharMingen, San Diego, CA, USA), MSH2 (clone FE11, diluted 1:50, Oncogene Research Products, Cambridge, MA, USA), MSH6 (clone GRBP. P1/2.D4, diluted 1:200; Serotec, Raleigh, NC, USA), and PMS2 (clone A16-4, diluted 1:200, BD PharMingen, San Diego, CA, USA). Non-neoplastic colonic mucosa and colorectal carcinoma known to be deficient of MLH1, MSH2, MSH6 and PMS2 were used as external positive and negative controls, respectively. Negative protein expression was defined as complete absence of nuclear staining within tumor cells in the presence of concurrent positive labeling in internal non-neoplastic tissues. Negative or equivocal protein expression detected on tissue microarray was followed up with immunohistochemistry performed on corresponding whole-tumor sections of the resection specimen following the same protocol. For the cases where repeated immunohistochemical staining was performed on whole sections, the results of the whole section were used for final analysis.

\section{Statistical Analysis}

Descriptive and comparative statistics were performed using IBM Statistical Software for the Social Sciences version 22 (IBM Corporation, Armonk, NY, USA). Continuous variables were compared using the Student's $t$-test. Categorical variables were compared using $\chi^{2}$ or the Fisher exact test depending on the number of observations. A $P$-value $<0.05$ was considered significant. Survival distributions were estimated using the Kaplan-Meier method and differences were assessed using the log-rank statistic. Time to event was calculated from date of colonic resection to date of event. An event for recurrence-free survival was defined as recurrence or death. Patients without the event of interest at last follow-up were censored. A Cox regression model, employing the backwards conditional method, was used to adjust for confounders. Parameters with $P<0.05$ on univariate analysis were included in the regression model.

\section{Results}

\section{Patient Demographics}

A total of 395 patients were included in the study. The average age of the patients at surgical resection 
Table 1 Patient demographics $(N=395)$

\begin{tabular}{|c|c|}
\hline & $\mathrm{n}^{\mathrm{a}}(\%)$ \\
\hline Age (years, mean \pm s.d. (range)) & $55 \pm 15(18-90)$ \\
\hline \multicolumn{2}{|l|}{ Sex } \\
\hline Male & $201(51)$ \\
\hline Female & $194(50)$ \\
\hline \multicolumn{2}{|l|}{ Tumor differentiation } \\
\hline Well & $11(3)$ \\
\hline Moderate & $326(83)$ \\
\hline Poor & $54(14)$ \\
\hline \multicolumn{2}{|l|}{ Medullary features } \\
\hline Yes & $16(7)$ \\
\hline No & $230(93)$ \\
\hline \multicolumn{2}{|l|}{ Signet ring cell features } \\
\hline Yes & $5(1)$ \\
\hline No & $371(99)$ \\
\hline \multicolumn{2}{|l|}{ Mucinous features } \\
\hline Yes & $69(18)$ \\
\hline No & $307(82)$ \\
\hline \multicolumn{2}{|l|}{ Location } \\
\hline Right colon & $155(39)$ \\
\hline Left colon & $239(61)$ \\
\hline \multicolumn{2}{|l|}{ Neoadjuvant treatment } \\
\hline Yes & $39(10)$ \\
\hline No & $343(90)$ \\
\hline \multicolumn{2}{|l|}{$T$-stage } \\
\hline Tis & $2(1)$ \\
\hline $\mathrm{T} 1$ & $28(7)$ \\
\hline $\mathrm{T} 2$ & $64(17)$ \\
\hline T3 & $220(57)$ \\
\hline $\mathrm{T} 4$ & $70(18)$ \\
\hline \multicolumn{2}{|l|}{$N$-stage } \\
\hline No & $188(50)$ \\
\hline N1 & $118(32)$ \\
\hline $\mathrm{N} 2$ & $67(18)$ \\
\hline \multicolumn{2}{|l|}{ AJCC stage } \\
\hline I & $60(15)$ \\
\hline II & $114(29)$ \\
\hline III & $132(34)$ \\
\hline IV & $83(21)$ \\
\hline \multicolumn{2}{|l|}{ Tumor-infiltrating lymphocyte score } \\
\hline $0-<1$ TIL per 10 high power fields & 74 (19) \\
\hline $1-1$ to 15 TILs per 10 high power fields & $175(46)$ \\
\hline $2->15$ but $<215$ TILs per 10 high power fields & $109(28)$ \\
\hline $3->215$ TILs per 10 high power fields & $35(9)$ \\
\hline \multicolumn{2}{|l|}{ Peritumoral lymphocyte aggregates } \\
\hline 0 - None & $230(59)$ \\
\hline $1-$ Few & $160(41)$ \\
\hline 2 - Many & $1(0)$ \\
\hline \multicolumn{2}{|l|}{ Mismatch-repair protein status } \\
\hline Mismatch-repair proficient & $321(83)$ \\
\hline Mismatch-repair deficient & $68(17)$ \\
\hline \multicolumn{2}{|l|}{ PD-1 expression (on TILs) } \\
\hline Low & $316(81)$ \\
\hline High & 76 (19) \\
\hline \multicolumn{2}{|l|}{ PD-L1 expression } \\
\hline Low & 375 (95) \\
\hline High & $19(5)$ \\
\hline Follow-up (months, mean \pm s.d. (range)) & $55 \pm 40(<1-393)$ \\
\hline
\end{tabular}

Abbreviations: AJCC, American Joint Committee on Cancer; PD-1, programmed death-1; PD-L1, programmed death-ligand 1; TILs, tumorinfiltrating lymphocytes.

${ }^{\mathrm{a} D i s c r e p a n c y}$ in the number of cases is due to lack of data on several cases. was $55 \pm 15$ years. Of the patients assessed, $55 \%$ were high stage (AJCC stage III or IV) and $10 \%$ received neoadjuvant chemotherapy. The complete patient demographics are shown in Table 1.

\section{Immunohistochemical Expression of PD-1, PD-L1, and Mismatch-Repair Proteins}

Data on PD-1 and PD-L1 were obtained on 389 tumors. PD-1 positivity was observed in both tumorinfiltrating lymphocytes and stromal lymphocytes. PD-L1 staining was observed in tumor cells as well as lymphocytes or immune cells; tumor cell staining for PD-L1 occurred in two patterns: primarily along the tumor-stromal interface $(84 \%$, Figure 1f) or diffuse (16\%, Figure $1 \mathrm{~g})$. In the formal scenario, the stained interfaces were not limited to the invasive front or the periphery of large groups of tumor cells; they could be intra-tumoral as well (wherever tumor cells were juxtaposed with stroma cells). For both markers, both membranous and cytoplasmic staining were regarded as positive.

Of the 389 tumors, 68 were found to be mismatchrepair deficient by immunohistochemistry. Mismatch-repair deficiency correlated significantly with PD-1 and PD-L1 expression by both staining intensity and the percentage of cell staining $(P<0.001$ for both). By maximizing the sensitivity and specificity of PD-L1 or PD-1 expression in predicting mismatchrepair status through receiver-operating characteristic curve analyses, ${ }^{15}$ we derived the following criteria to separate tumors into high and low PD-L1 and PD-1 expression categories. 'High-level PD-L1' corresponded to $>1 \%$ of tumor cells staining with 2+ intensity; 'high PD-1-positive tumor-infiltrating lymphocytes' corresponded to a measure of $>1.43$ tumor-infiltrating lymphocytes of $1+$ or $2+$ intensity per square millimeter. With these criteria, 18 and $50 \%$ of mismatch-repair deficient tumors exhibited high-level PD-L1 and high PD-1-positive tumor-infiltrating lymphocytes, respectively; on the other hand, these rates for mismatch-repairproficient tumors were only $2 \%$ and $13 \%$, respectively $(P<0.001$ for both).

For the entire group, 5\% had high-level PD-L1 (19/394) and 19\% had high PD-1-positive tumor-infiltrating lymphocytes (76/392). The PD-L1 staining was diffuse in $16 \%$ of the high-level positive cases. Expression of PD-L1 and that of PD-1 were positively correlated: $68 \%$ of the cases with high-level PD-L1 also displayed high PD-1 positive tumor-infiltrating lymphocytes, and 98\% of the cases with low PD-1 positive tumor-infiltrating lymphocytes also had low-level PD-L1 $(r=0.28$, $P<0.001)$. PD-1 levels maintained a positive correlation with PD-L1 levels when assessing only the mismatch-repair deficient or mismatch-repair proficient tumors $(P=0.017$ and $P=0.013$, respectively). 
Association Between PD-L1/PD-1 and Clinicopathologic Parameters

The association between PD-1 expression, PD-L1 expression, and clinicopathologic parameters is shown in Table 2. High PD-1-positive tumor-infiltrating lymphocytes and high-level PD-L1 correlated with the various clinicopathologic parameters that were more often associated with mismatch-repair deficiency including medullary morphology, right sided location, high H\&E tumor-infiltrating lymphocyte score, high H\&E peritumoral lymphocyte aggregate score, and younger age (all $P<0.05$ ). Many of these associations remained significant even when controlling for mismatch-repair status, including medullary morphology, high H\&E tumor-infiltrating lymphocyte score, and high H\&E peritumoral lymphocyte aggregate score (all $P<0.05$ ). Overall, highlevel PD-L1 expression was also associated with female sex $(P=0.029)$, whereas high PD-1-positive tumor-infiltrating lymphocytes was associated with lower $\mathrm{N}$-stage $(P=0.001)$ and lower overall AJCC stage group $(P<0.001)$.

\section{Survival Analysis}

The follow-up time for all patients was $55 \pm 40$ months (range, 0.4-393 months). Univariate analysis of the entire patient cohort identified the following parameters as significant predictors for recurrence-free survival: age $(P=0.041)$, sex $(P=0.025)$, T-stage $(P<0.001)$, N-stage $(P<0.001)$, AJCC stage group $(P<0.001), H \&$ E tumor-infiltrating lymphocyte score $(P=0.002)$, H\&E peritumoral lymphocyte aggregate score $(P=0.007)$, and PD-1 expression $(P=0.011)$.

When the patients were stratified by mismatchrepair status, many factors remained significant. However, the significance for PD-1 expression was only observed in the mismatch-repair-deficient group (Figure 2). High-level PD-1-positive tumorinfiltrating lymphocytes correlated with a significantly better recurrence-free survival among mismatch-repair-deficient patients (Figures 2c, $P=0.041$ ). In addition, as shown in Figure 2, tumor PD-L1 expression also emerged as a significant factor in the mismatch-repair-deficient group; high-level PD-L1 was associated with a significantly worse recurrence-free survival (Figures $2 f, P=0.026$ ). On the other hand, in the mismatch-repair-proficient group, neither PD-1 nor PD-L1 showed a statistically significant association with recurrence-free survival.

On multivariate analysis, both PD-1 and PD-L1 expression were shown to be independent factors associated with recurrence-free survival within the mismatch-repair-deficient group (PD-1: hazard ratio 0.03, 95\% confidence interval, 0.0-0.42, $P=0.01$; PD-L1: hazard ratio 22.86, 95\% confidence interval, 1.99-263.21, $P=0.012$ ), along with AJCC staging (hazard ratio 5.36, 95\% confidence interval, 1.81-15.84, $P=0.002$ ) (Table 3). PD-1 and PD-L1 were not independent factors associated with recurrence-free survival in the mismatch-repairproficient group or the entire patient cohort.

Further assessment revealed that within the mismatch-repair deficient group, PD-1 and PD-L1 were associated with recurrence-free survival in a co-dependent manner with combined high PD-L1 expression and high PD-1 positive tumor-infiltrating lymphocytes associating with a worse recurrencefree survival. As shown in Table 4, the association of high PD-1-positive tumor-infiltrating lymphocytes with a better recurrence-free survival was observed only among the group that had low-level PD-L1 $(P=0.006)$, and not among the group with highlevel PD-L1 $(P=0.061)$. Conversely, the association of high PD-L1 levels with a worse recurrence-free survival was observed only among the group that had high PD-1-positive tumor-infiltrating lymphocytes $(P<0.001)$ and not in the group with low PD-1positive tumor-infiltrating lymphocytes $(P=0.082)$.

\section{Discussion}

This study represents one of the first studies to systematically evaluate the frequency and pattern of immunohistochemical expression of PD-1 and PD-L1 in colorectal carcinoma. Our results showed that immunohistochemical detection of these markers was feasible and technically achievable. Of the 394 colorectal carcinomas that we evaluated, the rate of high PD-1-positive tumor-infiltrating lymphocytes was $19 \%$, and high-level PD-L1 expression in the tumor cells was 5\%. Both high PD-1-ositive tumorinfiltrating lymphocytes and high-level PD-L1 were significantly more frequent in mismatch-repairdeficient colorectal carcinomas than in mismatchrepair-proficient colorectal carcinomas. In addition, our results showed for the first time that the positive prognostic impact of tumor lymphocytes in patients with mismatch-repair-deficient colorectal carcinoma was negated by the presence of high-level PD-L1 in the tumors.

Defining the patterns of immunohistochemical expression of PD-1 and PD-L1 in colorectal carcinoma is clinically relevant as it provides a foundation for further exploration of the test's utility in predicting treatment response to immune checkpoint inhibition targeting the PD-1 pathway. In our analysis, we found immunohistochemical assessment of PD-1 to be a reliable and objective measure to evaluate the extent of PD-1 expression. For PD-L1 staining, we used a monoclonal antibody against PD-L1 from Cell Signaling (clone E1L3N), and observed two readily recognizable positive staining patterns in colorectal carcinoma tumor cells: diffuse and focal. The former is rare, constituting $16 \%$ of the positive cases. When the staining is focal, it is characteristically present along the tumor-stromal interface. In the positive cases, positive staining in background lymphocytes and mononuclear stromal cells frequently exists. 
Table 2 Comparison between PD-L1 high and low cases, and between PD-1 high and low cases ${ }^{\mathrm{a}}$

\begin{tabular}{|c|c|c|c|c|c|c|}
\hline & & $P D-L 1$ & & & PD-1 (on TILs) & \\
\hline & $\operatorname{Low}(N=375)$ & $\operatorname{High}(N=19)$ & & $\operatorname{Low}(N=316)$ & $\operatorname{High}(N=76)$ & \\
\hline & $N(\%)$ & $N(\%)$ & $\mathrm{P}$-value & $N(\%)$ & $N(\%)$ & $\mathrm{P}$-value \\
\hline Age (years, mean \pm s.d.) & $55 \pm 15$ & $65 \pm 15$ & 0.002 & $55 \pm 14$ & $59 \pm 16$ & 0.031 \\
\hline $\begin{array}{l}\text { Sex } \\
\text { M } \\
\text { F }\end{array}$ & $\begin{array}{l}195(52) \\
180(48)\end{array}$ & $\begin{array}{r}5(26) \\
14(74)\end{array}$ & 0.029 & $\begin{array}{l}163(52) \\
153(48)\end{array}$ & $\begin{array}{l}37(49) \\
39(51)\end{array}$ & 0.650 \\
\hline $\begin{array}{l}\text { Tumor differentiation } \\
\text { Well } \\
\text { Moderate } \\
\text { Poor }\end{array}$ & $\begin{aligned} 10 & (3) \\
314 & (85) \\
47 & (13)\end{aligned}$ & $\begin{aligned} 1 & (5) \\
11 & (58) \\
7 & (37)\end{aligned}$ & 0.008 & $\begin{aligned} 9 & (3) \\
267 & (85) \\
16 & (21)\end{aligned}$ & $\begin{aligned} & 2(3) \\
57 & (76) \\
37 & (12)\end{aligned}$ & 0.098 \\
\hline $\begin{array}{l}\text { Medullary features } \\
\text { Yes } \\
\text { No }\end{array}$ & $\begin{array}{c}10(4) \\
223(96)\end{array}$ & $\begin{array}{l}6(46) \\
7(54)\end{array}$ & $<0.001$ & $\begin{array}{c}5(3) \\
118(97)\end{array}$ & $\begin{array}{l}11(21) \\
41(79)\end{array}$ & $<0.001$ \\
\hline $\begin{array}{l}\text { Signet ring cell features } \\
\text { Yes } \\
\text { No }\end{array}$ & $\begin{array}{c}5(1) \\
353(99)\end{array}$ & $\begin{array}{c}0(0) \\
17(100)\end{array}$ & 1.000 & $\begin{array}{c}0(0) \\
296(98)\end{array}$ & $\begin{array}{c}0(0) \\
73(100)\end{array}$ & 0.588 \\
\hline $\begin{array}{l}\text { Mucinous features } \\
\text { Yes } \\
\text { No }\end{array}$ & $\begin{array}{r}65(18) \\
293(82)\end{array}$ & $\begin{array}{r}3(18) \\
14(82)\end{array}$ & 0.958 & $\begin{array}{r}57(19) \\
244(81)\end{array}$ & $\begin{array}{l}11(15) \\
62(85)\end{array}$ & 0.442 \\
\hline $\begin{array}{l}\text { Location } \\
\text { Right colon } \\
\text { Left colon }\end{array}$ & $\begin{array}{l}142(38) \\
232(62)\end{array}$ & $\begin{array}{r}12(63) \\
7(37)\end{array}$ & 0.028 & $\begin{array}{l}106(34) \\
209(66)\end{array}$ & $\begin{array}{l}49(64) \\
27(36)\end{array}$ & $<\mathbf{0 . 0 0 1}$ \\
\hline $\begin{array}{l}\text { Neoadjuvant treatment } \\
\text { Yes } \\
\text { No }\end{array}$ & $\begin{array}{r}39(11) \\
324(89)\end{array}$ & $\begin{array}{c}0(0) \\
18(100)\end{array}$ & 0.142 & $\begin{array}{r}36(12) \\
270(88)\end{array}$ & $\begin{array}{c}3(4) \\
71(96)\end{array}$ & 0.050 \\
\hline $\begin{array}{l}\text { T-Stage } \\
\text { Tis } \\
\text { T1 } \\
\text { T2 } \\
\text { T3 } \\
\text { T4 }\end{array}$ & $\begin{aligned} 2 & (1) \\
26 & (7) \\
59 & (16) \\
213 & (58) \\
65 & (18)\end{aligned}$ & $\begin{array}{l}0(0) \\
2(11) \\
5(28) \\
6(33) \\
5(28)\end{array}$ & 0.323 & $\begin{aligned} 2 & (1) \\
19 & (6) \\
50 & (16) \\
176 & (57) \\
61 & (20)\end{aligned}$ & $\begin{aligned} 0 & (0) \\
9 & (12) \\
14 & (19) \\
43 & (57) \\
9 & (12)\end{aligned}$ & 0.238 \\
\hline $\begin{array}{l}N \text {-stage } \\
\text { No } \\
\text { N1 } \\
\text { N2 }\end{array}$ & $\begin{array}{r}178(51) \\
109(31) \\
67(19)\end{array}$ & $\begin{array}{l}9(50) \\
9(50) \\
0(0)\end{array}$ & 0.067 & $\begin{array}{r}136(45) \\
105(35) \\
59(20)\end{array}$ & $\begin{array}{r}51(71) \\
13(18) \\
8(11)\end{array}$ & 0.001 \\
\hline $\begin{array}{l}\text { AJCC stage } \\
\text { I } \\
\text { II } \\
\text { III } \\
\text { IV }\end{array}$ & $\begin{array}{r}56(15) \\
110(30) \\
123(33) \\
80(22)\end{array}$ & $\begin{array}{l}4(21) \\
3(16) \\
9(47) \\
3(16)\end{array}$ & 0.397 & $\begin{aligned} 41 & (13) \\
82 & (26) \\
113 & (36) \\
75 & (24)\end{aligned}$ & $\begin{array}{c}19(25) \\
31(41) \\
19(25) \\
7(9)\end{array}$ & $<0.001$ \\
\hline $\begin{array}{l}\text { Tumor-infiltrating lymphocyte score } \\
0-<1 \text { TIL per } 10 \text { high power fields } \\
1-1 \text { to } 15 \text { TILs per } 10 \text { high power fields } \\
2->15 \text { but }<215 \text { TILs per } 10 \text { high power fields } \\
3->215 \text { TILs per } 10 \text { high power fields }\end{array}$ & $\begin{aligned} 71 & (19) \\
175 & (47) \\
101 & (27) \\
26 & (7)\end{aligned}$ & $\begin{array}{l}3(16) \\
0(0) \\
7(37) \\
9(47)\end{array}$ & $<0.001$ & $\begin{aligned} 69 & (22) \\
161 & (51) \\
76 & (24) \\
8 & (3)\end{aligned}$ & $\begin{array}{c}3(4) \\
13(17) \\
33(43) \\
27(36)\end{array}$ & $<0.001$ \\
\hline $\begin{array}{l}\text { Peritumoral lymphocyte aggregates } \\
\quad 0 \text { - None } \\
1 \text { - Few } \\
2 \text { - Many }\end{array}$ & $\begin{aligned} 225 & (61) \\
145 & (39) \\
1 & (0)\end{aligned}$ & $\begin{aligned} 4 & (21) \\
15 & (79) \\
0 & (0)\end{aligned}$ & 0.003 & $\begin{array}{c}203(65) \\
109(35) \\
1(0)\end{array}$ & $\begin{array}{c}24(32) \\
51(68) \\
0(0)\end{array}$ & $<\mathbf{0 . 0 0 1}$ \\
\hline $\begin{array}{l}\text { Mismatch-repair protein status } \\
\text { Mismatch-repair proficient } \\
\text { Mismatch-repair deficient }\end{array}$ & $\begin{array}{r}314(85) \\
55(15)\end{array}$ & $\begin{array}{r}7(37) \\
12(63)\end{array}$ & $<0.001$ & $\begin{array}{r}277(89) \\
34(11)\end{array}$ & $\begin{array}{l}41(55) \\
34(45)\end{array}$ & $<0.0001$ \\
\hline $\begin{array}{l}\text { PD-1 expression on TILs } \\
\text { Low } \\
\text { High }\end{array}$ & $\begin{array}{r}309(83) \\
63(17)\end{array}$ & $\begin{array}{r}6(32) \\
13(68)\end{array}$ & $<0.001$ & & & \\
\hline
\end{tabular}

Abbreviations: AJCC, American Joint Committee on Cancer; PD-1, programmed death-1; PD-L1, programmed death-ligand 1; TILs, tumorinfiltrating lymphocytes.

${ }^{a}$ Number of cases for each category may be less than the total due to lack of data on several cases. Statistically significant $P$-values $(P<0.05)$ are highlighted in bold. 
These patterns are similar to that observed in other cancer types where the utility of immunohistochemistry as predictive markers is better defined, including melanoma ${ }^{16}$ and lung carcinoma. ${ }^{17,18}$

In our study, we further demonstrated that, in colorectal carcinoma, the high expressers of both PD-1 and PD-L1 were mostly mismatch-repairdeficient tumors. Recently, molecular analyses have indicated that only a small subset of colorectal carcinomas has upregulated mRNA expression of the major immune checkpoint genes (including PDCD1, CD274, CTLA4, LAG3, and TIGIT) and this subset falls within the category of mismatch-repairdeficient tumors. ${ }^{19,20}$ Clinically, as reported by Le et al., ${ }^{10}$ in a phase II clinical trial, colorectal carcinoma patients that showed immune-related objective response to anti-PD-1 treatment all had mismatch-repair-deficient tumors. Thus, all evidence suggests the existence of a particular subset of colorectal carcinomas that are mismatch-repairdeficient, have activated PD-L1 function, and respond to immune checkpoint inhibition. However, as yet, data directly indicating that the positive cases by immunohistochemistry are indeed the cases with upregulated immune checkpoint genes and/or the cases respond to anti-PD-1 therapy are still lacking. Thus, further studies are needed. The PD-1 and PD-L1 immunohistochemical staining patterns observed in our study should serve as a foundation for such studies, and incorporation of immunohistochemistry in clinical trials is warranted.

Tumor-infiltrating lymphocytes are in general regarded as an indicator of host immune response to the tumor,11,21 and have long been recognized as a favorable prognostic marker in colorectal carcinoma. ${ }^{22-25}$ Prognostic studies thus far, however, have mostly evaluated tumor-infiltrating lymphocytes outside the context of immune checkpoints. In this study, we demonstrated that the expression of immune checkpoint receptor PD-1 and its ligand PD-L1 can indeed affect the impact of tumorinfiltrating lymphocytes on recurrence-free survival. Specifically, we demonstrated that overall, high PD-1-positive tumor-infiltrating lymphocytes were associated with a favorable outcome in mismatchrepair deficient colorectal carcinoma patients, but high-level PD-L1 expression was associated with a worse recurrence-free survival. More interestingly, we showed that when patients were further stratified by PD-L1 or PD-1 levels, the favorable association with outcomes of PD-1-positive tumor-infiltrating lymphocytes was only observed in group of tumors that did not have high-level PD-L1 expression. Tumors with high-level PD-L1 expression had poor prognosis regardless of PD-1 expression. Conversely, the association of high-level PD-L1 expression with worse recurrence-free survival was observed only in the group of tumors that had high PD-1-positive tumor-infiltrating lymphocytes. Tumors with low levels of PD-1 tumor-infiltrating lymphocytes had poor prognosis, regardless of PD-L1 expression.
Immune checkpoints represent the major defense system of the tumor against the host's antitumor immunity. ${ }^{26}$ Efficient checkpoint function of the tumor can lead to immune evasion and consequently tumor progression. As PD-1 is a major component of the PD-1 checkpoint pathway, our observation that high-level PD-1-positive tumor-infiltrating lymphocytes correlated with favorable recurrence-free survival in mismatch-repair-deficient colorectal carcinomas seems unexpected. However, the interpretation of our observation has to be made in conjunction with the fact that the frequency of high-level PD-L1 expression is very low in colorectal carcinoma. High tumor-infiltrating lymphocytes are common in mismatch-repair-deficient colorectal carcinomas (presumably secondary to neo-antigens generated by a high burden of frameshift mutations), ${ }^{27,28}$ and as an inherent component of this adaptive immunity that elicits the tumor-infiltrating lymphocytes, PD-1positive tumor-infiltrating lymphocytes develop as well. However, for PD-1 to exert its immuneinhibitory effect, there also needs to be PD-L1 expression by the tumor cells or other immune cells. Thus, given the low frequency of high PD-L1 expression in colorectal carcinoma, the high PD-1positive tumor-infiltrating lymphocytes we observed in most cases may merely reflect an elevation of effector $\mathrm{T}$ cells in general. This assumption is indeed supported by the fact that, when we sub-stratified our cases, the favorable survival associated with high PD-1-positive tumor-infiltrating lymphocytes is only maintained in tumors with no or low PD-L1, and not in tumors with high PD-L1, and the negative association with recurrence-free survival of PD-L1 was no longer significant when the tumors did not have high levels PD-1-positive tumor-infiltrating lymphocytes.

Thus, mismatch-repair-deficient colorectal carcinomas with 'high PD-1 and no or low PD-L1' are likely to have an anti-tumoral immune microenvironment in which the cytotoxic tumor-infiltrating lymphocytes prevails and consequently they are likely to have a better prognosis, whereas those with both 'high PD-1 and high PD-L1' are likely to harbor ongoing immune checkpoint activity and are consequently more prone to progression and poor prognosis. It can be further inferred that in colorectal carcinoma, particularly in those that are mismatchrepair-deficient, tumor-infiltrating lymphocytes are necessary for both anti-tumoral and pro-tumoral immunity. The balance between the two opposing immune functions is largely dependent on the level of PD-L1 expression. Further exploration in this area may shed light not only on the understanding of PD-1-positive tumor-infiltrating lymphocytes as a prognostic indicator, but also on the utility of PD-1 and PD-L1 expression as predictors for treatment response to immune therapy. Colorectal carcinomas with both 'high PD-1 and PD-L1' may have an active immune checkpoint activity and may therefore represent the subset that benefit from anti-PD-1 therapy. 
a

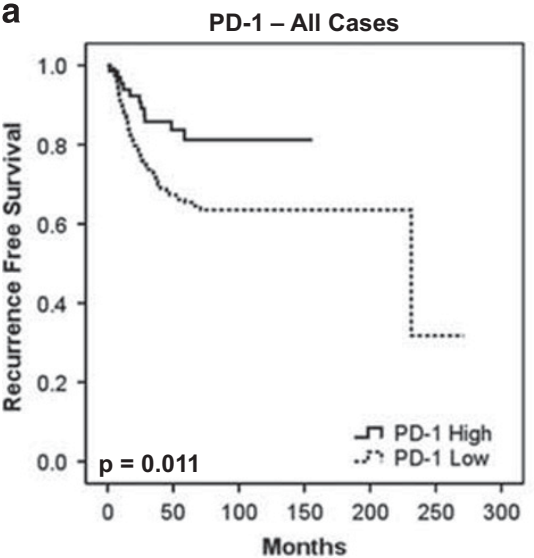

d

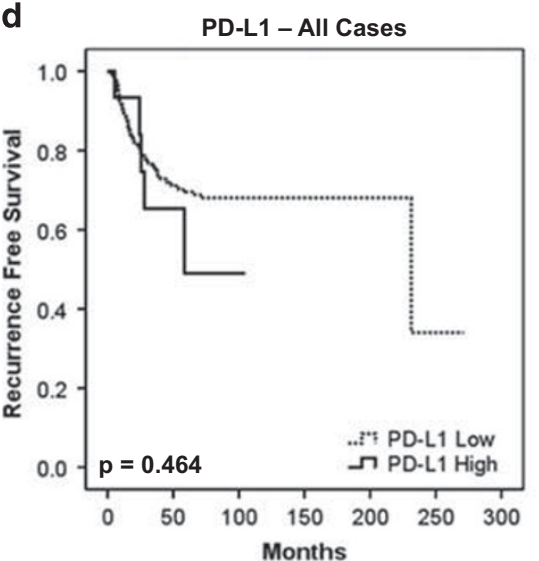

b

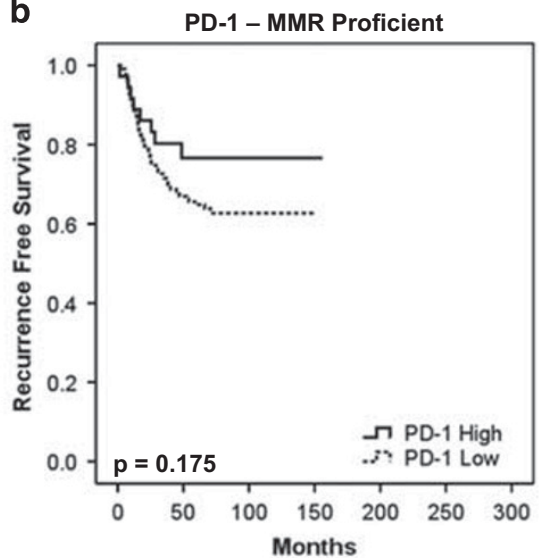

e

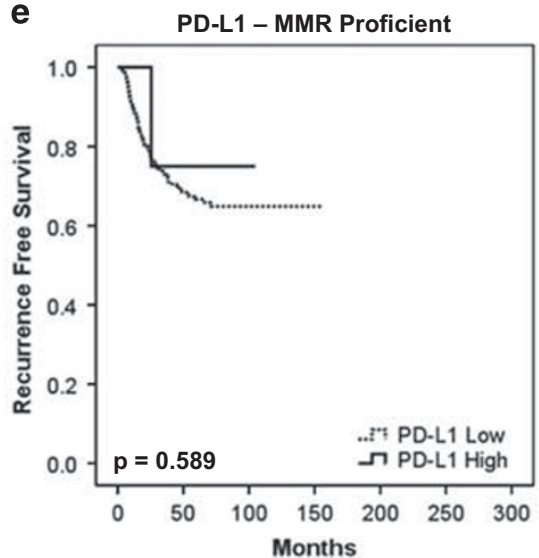

C

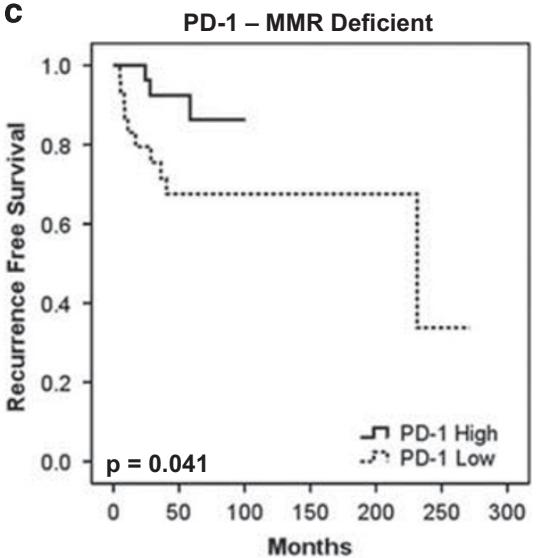

f

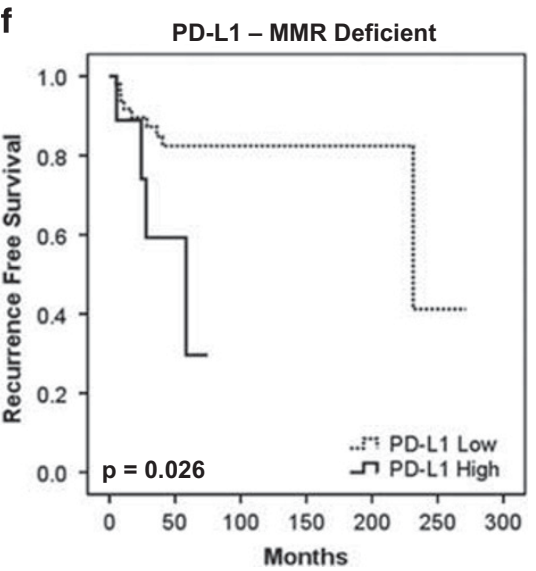

Figure 2 Kaplan-Meier survival curves depicting recurrence-free survival of patients with colorectal carcinoma stratified by tumorinfiltrating lymphocyte PD-1 expression and tumor cell PD-L1 expression. PD-1 expression stratified survival when all patients were assessed (a). It was not a significant prognostic factor in patients with mismatch-repair proficient carcinomas (b), but was significant among mismatch-repair-deficient carcinomas (c). PD-L1 expression did not stratify survival when assessing all patients (d) or in patients with mismatch-repair proficient carcinomas (e), but did significantly stratify patients with mismatch-repair deficient carcinomas (f). $P$-values were calculated by log-rank test.

Table 3 Relationship between clinicopathological characteristics and RFS among mismatch-repair-deficient tumors

\begin{tabular}{|c|c|c|c|c|c|}
\hline & \multicolumn{3}{|c|}{ Univariate analysis } & \multicolumn{2}{|c|}{ Multivariate analysis } \\
\hline & Hazard ratio & P-value (Cox) & P-value (log-rank) & Hazard ratio & $\mathrm{P}$-value \\
\hline Age & $0.98(0.95-1.01)$ & 0.256 & & - & - \\
\hline Sex & $1.16(0.37-3.59)$ & 0.801 & 0.801 & - & - \\
\hline Tumor differentiation & $1.32(0.48-3.63)$ & 0.587 & 0.525 & - & - \\
\hline Medullary features & $1.67(0.36-7.72)$ & 0.514 & 0.510 & - & - \\
\hline Signet ring cell features & No events & & & - & - \\
\hline Mucinous features & $1.18(0.35-3.91)$ & 0.793 & 0.793 & - & - \\
\hline Tumor location & $0.26(0.07-0.91)$ & 0.034 & 0.026 & NS & NS \\
\hline Neoadjuvant treatment & No events & & & - & - \\
\hline T-Stage & $3.42(1.29-9.07)$ & 0.014 & 0.073 & NS & NS \\
\hline N-Stage & $2.49(1.23-5.07)$ & 0.012 & 0.023 & NS & NS \\
\hline AJCC Stage & $5.01(2.43-10.35)$ & $<0.001$ & $<0.001$ & $5.36(1.81-15.84)$ & 0.002 \\
\hline Tumor-infiltrating lymphocyte score & $0.49(0.27-0.90)$ & 0.021 & 0.077 & NS & NS \\
\hline Peritumoral lymphocyte aggregates & $0.87(0.26-2.88)$ & 0.813 & 0.812 & - & - \\
\hline PD-1 expression on tumor-infiltrating lymphocytes & $0.28(0.08-1.03)$ & 0.056 & 0.041 & $0.03(0.00-0.42)$ & 0.010 \\
\hline PD-L1 expression on tumor & $3.60(1.08-12.0)$ & 0.037 & 0.026 & $22.86(1.99-263.21)$ & 0.012 \\
\hline
\end{tabular}

Abbreviations: AJCC, American Joint Committee on Cancer; N, node; NS, not significant; PD-1, programmed death-1; PD-L1, programmed deathligand 1; T, tumor.

Statistically significant $P$-values $(P<0.05)$ are highlighted in bold. 
Table 4 Interaction between PD-L1 and PD-1 on RFS among mismatch-repair-deficient tumors

\begin{tabular}{|c|c|c|c|}
\hline & & 5 -year RFS (95\% CI) & $\mathrm{P}$-value \\
\hline Low-level PD-L1 & $\begin{array}{l}\text { Low PD-1-positive TILs } \\
\text { High PD-1-positive TILs }\end{array}$ & $\begin{array}{c}68 \%(50 \%-87 \%) \\
\text { All cases censored }\end{array}$ & 0.006 \\
\hline High-level PD-L1 & $\begin{array}{l}\text { Low PD-1-positive TILs } \\
\text { High PD-1-positive TILs }\end{array}$ & $\begin{array}{l}50 \%(0 \%-100 \%) \\
33 \%(0 \%-83 \%)\end{array}$ & 0.061 \\
\hline Low PD-1-positive TILs & $\begin{array}{l}\text { Low-level PD-L1 } \\
\text { High-level PD-L1 }\end{array}$ & $\begin{array}{l}68 \%(50 \%-87 \%) \\
50 \%(0 \%-100 \%)\end{array}$ & 0.082 \\
\hline High PD-1-positive TILs & $\begin{array}{l}\text { Low-level PD-L1 } \\
\text { low-level PD-L1 }\end{array}$ & $\begin{array}{l}\text { All cases censored } \\
33 \%(0 \%-83 \%)\end{array}$ & $<0.001$ \\
\hline
\end{tabular}

Abbreviations: CI, confidence interval; PD-1, programmed death-1; PD-L1, programmed death-ligand 1; RFS, recurrence-free survival; TILs, tumorinfiltrating lymphocytes.

$P$-value calculated by log-rank test, with statistically significant differences $(P<0.05)$ in bold.

A limitation of our study is that the assessment of PD-1 and PD-L1 was performed on tissue microarray only. As the staining of these markers may be focal and heterogeneous, tissue array cores may under-represent cases with focal positivity. Furthermore, even though our overall case number is relatively large, the positive events both in terms of positive PD-1/PD-L1 labeling and clinical outcome are still small.

Nonetheless, our study provided data indicating that PD-1 and PD-L1 immunohistochemistry is feasible in colorectal carcinoma, and in this tumor type, high-level expression of these markers is a feature tightly associated with mismatch-repair deficiency. Our results may serve as a foundation to further explore whether there is indeed added value of PD-1/PD-L1 immunohistochemistry to MSI in selecting colorectal carcinoma that might respond to anti-PD-1 therapy. Furthermore, our data indicated that immunohistochemical detection of PD-1 and PD-L1 has the potential to refine our understanding of the prognostic value of tumor-infiltrating lymphocytes in colorectal carcinoma; PD-L1 expression may allow the identification of the mismatchrepair-deficient colorectal cancers that have a poor prognosis despite having high-level tumor-infiltrating lymphocytes.

\section{Acknowledgments}

This research was supported in part by the NIH/NCI Cancer Center Support Grant P30 CA008748.

\section{Disclosure/conflict of interest}

The authors declare no conflict of interest.

\section{References}

1 Hamid O, Robert C, Daud A et al. Safety and tumor responses with lambrolizumab (anti-PD-1) in melanoma. N Engl J Med 2013;369:134-144.
2 Herbst RS, Soria JC, Kowanetz M et al. Predictive correlates of response to the anti-PD-L1 antibody MPDL3280A in cancer patients. Nature 2014;515: 563-567.

3 Powles T, Eder JP, Fine GD et al. MPDL3280A (anti-PDL1) treatment leads to clinical activity in metastatic bladder cancer. Nature 2014;515:558-562.

4 Topalian SL, Hodi FS, Brahmer JR et al. Safety, activity, and immune correlates of anti-PD-1 antibody in cancer. N Engl J Med 2012;366:2443-2454.

5 Brahmer JR, Tykodi SS, Chow LQM et al. Safety and activity of anti-PD-L1 antibody in patients with advanced cancer. N Engl J Med 2012;366: 2455-2465.

6 Topalian SL, Sznol M, McDermott DF et al. Survival, durable tumor remission, and long-term safety in patients with advanced melanoma receiving nivolumab. J Clin Oncol 2014;32:1020-1030.

7 Sunshine J, Taube JM. PD-1/PD-L1 inhibitors. Curr Opin Pharmacol 2015;23:32-38.

8 Taube JM, Klein A, Brahmer JR et al. Association of PD-1, PD-1 ligands, and other features of the tumor immune microenvironment with response to anti-PD-1 therapy. Clin Cancer Res 2014;20:5064-5074.

9 Brahmer JR, Drake CG, Wollner I et al. Phase I study of single-agent anti-programmed death-1 (MDX-1106) in refractory solid tumors: safety, clinical activity, pharmacodynamics, and immunologic correlates. J Clin Oncol 2010;28:3167-3175.

10 Le DT, Uram JN, Wang $\mathrm{H}$ et al. PD-1 Blockade in Tumors with Mismatch-Repair Deficiency. N Engl J Med 2015;372:2509-2520.

11 Mantovani A, Romero P, Palucka AK et al. Tumour immunity: effector response to tumour and role of the microenvironment. Lancet 2008;371:771-783.

12 Shia J, Ellis NA, Paty PB et al. Value of histopathology in predicting microsatellite instability in hereditary nonpolyposis colorectal cancer and sporadic colorectal cancer. Am J Surg Pathol 2003;27:1407-1417.

13 Goldstein J, Tran B, Ensor J et al. Multicenter retrospective analysis of metastatic colorectal cancer (CRC) with high-level microsatellite instability (MSI-H)Ann Oncol 2014;25:1032-1038.

14 Nordholm-Carstensen A, Krarup P-M, Morton D et al. Danish Colorectal Cancer Group. Mismatch repair status and synchronous metastases in colorectal cancer: 
a nationwide cohort study. Int J Cancer 2015;137: 2139-2148.

15 Perkins NJ, Schisterman EF. The inconsistency of "Optimal" cut-points using two ROC based criteria. Am J Epidemiol 2006;163:670-675.

16 Madore J, Vilain RE, Menzies AM et al. PD-L1 expression in melanoma shows marked heterogeneity within and between patients: implications for antiPD-1/PD-L1 clinical trials. Pigment Cell Melanoma Res 2015;28:245-253.

17 Garon EB, Rizvi NA, Hui R et al. Pembrolizumab for the treatment of non-small-cell lung cancer. N Engl J Med 2015;372:2018-2028.

18 Passiglia F, Bronte G, Bazan V et al. PD-L1 expression as predictive biomarker in patients with NSCLC: a pooled analysis. Oncotarget 2016; e-pub ahead of print.

19 Shia J, Kuk D, Schultz N et al. Comprehensive morphological assessment of colorectal cancers characterized by The Cancer Genome Atlas (TCGA) identifies specific morphology-molecular alteration associations [abstract]. Mod Pathol 2016;29(Suppl 2):200A.

20 Lal N, Beggs AD, Willcox BE et al. An immunogenomic stratification of colorectal cancer: implications for development of targeted immunotherapy. Oncoimmunology 2015;4:e976052.

21 Pagès F, Berger A, Camus $M$ et al. Effector memory $\mathrm{T}$ cells, early metastasis, and survival in colorectal cancer. N Engl J Med 2005;353:2654-2666.
22 Klintrup K, Mäkinen JM, Kauppila S et al. Inflammation and prognosis in colorectal cancer. Eur J Cancer 2005;41:2645-2654.

23 Roxburgh CSD, Salmond JM, Horgan PG et al. Comparison of the prognostic value of inflammation-based pathologic and biochemical criteria in patients undergoing potentially curative resection for colorectal cancer. Ann Surg 2009;249:788-793.

24 Morris M, Platell C, Iacopetta B. Tumor-infiltrating lymphocytes and perforation in colon cancer predict positive response to 5-fluorouracil chemotherapy. Clin Cancer Res 2008;14:1413-1417.

25 Ropponen KM, Eskelinen MJ, Lipponen PK et al. Prognostic value of tumour-infiltrating lymphocytes (TILs) in colorectal cancer. J Pathol 1997;182:318-324.

26 Pardoll DM. The blockade of immune checkpoints in cancer immunotherapy. Nat Rev Cancer 2012;12: 252-264.

27 Llosa NJ, Cruise M, Tam A et al. The vigorous immune microenvironment of microsatellite instable colon cancer is balanced by multiple counter-inhibitory checkpoints. Cancer Discov 2015;5:43-51.

28 Smyrk TC, Watson P, Kaul K et al. Tumor-infiltrating lymphocytes are a marker for microsatelliteinstability in colorectal carcinoma. Cancer 2001;91: 2417-2422. 